\title{
MESH INDEPENDENCE OF MATRIX-FREE METHODS FOR PATH FOLLOWING*
}

\author{
W. R. FERNG ${ }^{\dagger}$ AND C. T. KELLEY ${ }^{\ddagger}$
}

\begin{abstract}
In this paper we consider a matrix-free path following algorithm for nonlinear parameter-dependent compact fixed point problems. We show that if these problems are discretized so that certain collective compactness and strong convergence properties hold, then this algorithm can follow smooth folds and capture simple bifurcations in a mesh independent way.
\end{abstract}

Key words. Path following, bifurcation, collective compactness, fold point, mesh independence, matrix-free method, GMRES, Arnoldi method, singularity

AMS subject classifications. 45G10, 65F10, 65H10, 65H17, 65J15

1. Introduction. The purpose of this paper is to extend the results in [4], [5], and [16] on mesh-independent convergence of the GMRES, [21], iterative method for linear equations to a class of matrix-free methods for solution of parameter dependent nonlinear equations of the form

$$
G(u, \lambda)=0 .
$$

In (1.1) $G: X \times \mathbb{R} \rightarrow X$ where $X$ is a Banach space.

We present an algorithm for numerical path following and detection of simple bifurcations together with conditions on a sequence of approximate problems,

$$
G^{h}(u, \lambda)=0,
$$

(with $G^{0}=G$ for consistency of notation) that imply that the performance of the algorithm is independent of the level $h$ of the discretization. Hence, for such problems, methods, and discretizations, the difficulties raised in [23] and [24] will not arise.

In this section we set the notation and specify the kinds of singlarities that we will consider. This discussion, and that of algorithms for path following in $\S 2.1$ and detection of simple bifurcation and branch switching in $\S 3$, do not depend on the discretization and we use the notation $G$ for both $G^{0}$ and $G^{h}$ for $h>0$. When we describe our assumptions on the discretization in $\S 4$ and present an example in $\S 5$, the distinction between $G^{0}$ and $G^{h}$ becomes important and the notation in those sections reflects that difference.

\subsection{Notation and Simple Singularities. We let}

$$
\Gamma(\lambda)=\{(u, \lambda) \mid G(u, \lambda)=0, \lambda \in \mathbb{R}\}
$$

denote the solution path in $X \times \mathbb{R}$ of pairs $(u, \lambda)$ that satisfy (1.1), $G_{u}$ denotes the Fréchet derivative with respect to $u$, and $G_{\lambda}$ the derivative with respect to the scalar $\lambda$.

\footnotetext{
*Version of May 19, 1998.

${ }^{\dagger}$ Department of Applied Mathematics, National Chiao-Tung University, Hsin-Chu 30050, Taiwan. (ferng@math.nctu.edu.tw). The research of this author was supported by National Science Council, Taiwan.

$\ddagger$ North Carolina State University, Department of Mathematics and Center for Research in Scientific Computation, Box 8205, Raleigh, N. C. 27695-8205 (Tim_Kelley@ncsu.edu). The research of this author was supported by National Science Foundation grant \#DMS-9700569.
} 
Throughout this paper we make

ASSUMPTION 1.1. $G$ is continuously Fréchet differentiable in $(u, \lambda) . G_{u}$ is a Fredholm operator of index zero, and

$$
I-G_{u}(u, \lambda), G_{\lambda} \in \mathcal{C O} \mathcal{M}(X) \text { for all }(u, \lambda) \in X \times \mathbb{R} \text {. }
$$

Here $\mathcal{C O} \mathcal{M}$ denotes the space of compact operators.

For $A \in \mathcal{L}(X)$, the space of bounded operators on $X$, we let $\mathcal{N}(A)$ be the null space of $A$ and $\mathcal{R}(A)$ the range of $A$. Assumption 1.1 implies that $\mathcal{R}\left(G_{u}(u, \lambda)\right)$ is closed in $X$ and the dimension of $\mathcal{N}\left(G_{u}(u, \lambda)\right)$ is the co-dimension of $\mathcal{R}\left(G_{u}(u, \lambda)\right)$.

Following [14], we say that a point $(u, \lambda) \in \Gamma$

- is a regular point if $G_{u}(u, \lambda)$ is nonsingular,

- is a simple singularity if 0 is an eigenvalue of $G_{u}$ with algebraic and geometric multiplicity one,

- is a simple fold if it is a simple singularity and $G_{\lambda} \notin \mathcal{R}\left(G_{u}\right)$.

- is a simple bifurcation point if it is a simple singularity and $G_{\lambda} \in \mathcal{R}\left(G_{u}\right)$.

2. Algorithms for Path Following and Branch Switching. In this section we describe the iterative methods for path following, detection of bifurcation, and branch switching that we analyze in the subsequent sections and discuss some alternative approaches. Our approach is matrix-free, which means that we use no matrix storage or matrix factorizations at all. However, there are many related methods to which our mesh-independence analysis is applicable. We refer the reader to [13] and [7] for other methods for path following and branch switching and to [20], [25], and [10] for iterative methods for detection of bifurcation.

2.1. Path Following and Arclength Continuation. For path following in the absence of singularities the standard approach is a predictor-corrector method. The methods differ in the manner in which $u^{\prime}(\lambda)=d u / d \lambda$ is computed. Assume that $\left(u_{0}, \lambda_{0}\right)$ is a known solution point and we want to compute $\left(u_{1}, \lambda_{1}\right)$ at a nearby $\lambda=\lambda_{1}$.

If the Jacobian $G_{u}\left(u_{0}, \lambda_{0}\right)$ is nonsingular, then the implicit function theorem insures the existence of a unique smooth arc of solutions $(u(\lambda), \lambda)$, through $\left(u_{0}, \lambda_{0}\right)$ with $u\left(\lambda_{0}\right)=u_{0}$. Furthermore, with the smoothness assumption on $G$, it follows that $u^{\prime}(\lambda)=\frac{d}{d \lambda} u(\lambda)$ exists and differentiation of (1.1) with respect to $\lambda$ gives the equation for $u^{\prime}$.

$$
G_{u}\left(u_{0}, \lambda_{0}\right) u^{\prime}\left(\lambda_{0}\right)=-G_{\lambda}\left(u_{0}, \lambda_{0}\right)
$$

The Euler predictor uses $u^{\prime}$ directly and approximates $u_{1}=u\left(\lambda_{1}\right)$ with

$$
u_{1}^{(0)}=u_{0}+\left(\lambda_{1}-\lambda_{0}\right) u^{\prime}\left(\lambda_{0}\right) .
$$

While the results in this paper can be applied to the solution of (2.1) by GMRES, we choose to avoid the addtional solve and approximate $u^{\prime}$ with a difference. The secant predictor is

$$
u_{1}^{(0)}=u_{0}+\frac{\lambda_{1}-\lambda_{0}}{\lambda_{0}-\lambda_{-1}}\left(u_{0}-u_{-1}\right)
$$

where $\left(u_{-1}, \lambda_{-1}\right)$ is a second solution pair with $\lambda_{-1}<\lambda_{0}$. 
The nonlinear iteration is an inexact Newton iteration [6] in which

$$
u_{1}^{k+1}=u_{1}^{k}+d^{k},
$$

where $d^{k}$ satisfies the inexact Newton condition

$$
\left\|G_{u}\left(u_{1}^{k}, \lambda_{1}\right) d^{k}+G\left(u_{1}^{k}, \lambda_{1}\right)\right\|<\eta_{k}\left\|G\left(u_{1}^{k}, \lambda_{1}\right)\right\| .
$$

The inexact Newton condition can be viewed as a relative residual termination criterion for an iterative linear solver applied to the equation for the Newton step

$$
G_{u}\left(u_{1}^{k}, \lambda_{1}\right) d=-G\left(u_{1}^{k}, \lambda_{1}\right) .
$$

When GMRES is used as the linear iteration, as it is in this paper, the nonlinear solver is referred to as Newton-GMRES.

The same procedure can be used if there are simple folds if the problem is expanded by using pseudo-arclength continuation, [12], [13]. Here we introduce a new parameter $s$ and solve

$$
F(x, s)=\left[\begin{array}{c}
G(x) \\
N(x, s)
\end{array}\right]=0,
$$

where $x=(u, \lambda)$ and $N(u, \lambda, s)=0$ is a normalization equation.

We will use the secant normalization

$$
\begin{aligned}
N(u, \lambda, s)= & \theta\left(\frac{u\left(s_{0}\right)-u\left(s_{-1}\right)}{s_{0}-s_{-1}}\right)^{T}\left(u(s)-u\left(s_{0}\right)\right) \\
& +(1-\theta)\left(\frac{\lambda\left(s_{0}\right)-\lambda\left(s_{-1}\right)}{s_{0}-s_{-1}}\right)\left(\lambda(s)-\lambda\left(s_{0}\right)\right)-\left(s-s_{0}\right)=0,
\end{aligned}
$$

when two points on the path are available and the norm-based normalization

$$
N(u, \lambda, s)=\theta\left\|u-u\left(s_{0}\right)\right\|^{2}+(1-\theta)\left|\lambda-\lambda\left(s_{0}\right)\right|^{2}-\left(s-s_{0}\right)^{2}
$$

to begin the path following. Both normalizations are independent of the discretization. This independence plays a role in the analyis that follows.

It is known, [14], that arclength continuation turns simple folds in $(u, \lambda)$ to regular points in $(u, \lambda, s)$.

3. Singular Point Detection and Branch Switching. Although a continuation procedure incorporated with a pseudo-arclength normalization can circumvent the computational difficulties caused by turning points and usually jump over bifurcation points, it is usually desirable and important to be able to detect and locate the singularities in both cases. In the case where direct methods are used for linear algebra a sign change in the determinant of $F$ can be used to detect simple bifurcation and a change in sign of $d \lambda / d s$ to detect simple folds. We use a variation of the approach in [10], which requires only the largest (in magnitude) solution of a generalized eigenvalue problem.

Suppose that $\left(x\left(s_{a}\right), s_{a}\right)$ and $\left(x\left(s_{b}\right), s_{b}\right)$ are two regular points and the path following procedure is going from $s_{a}$ to $s_{b}$. Let $A\left(s_{a}\right)=F_{x}\left(x\left(s_{a}\right), s_{a}\right)$ and $A\left(s_{b}\right)=F_{x}\left(x\left(s_{b}\right), s_{b}\right)$. Then both 
$A\left(s_{a}\right)$ and $A\left(s_{b}\right)$ are nonsingular. Recall that if $(x(s), s)$ is a bifurcation point on the solution path, then the null space $\mathcal{N}\left(F_{x}(x(s), s)\right)$ is one dimensional and there exists a nonzero vector $v$ such that

$$
F_{x}(x(s), s) v=0
$$

Applying the Lagrange interpolation to $A(s)=F_{x}(x(s), s)$ we have

$$
A(s)=\frac{s-s_{b}}{s_{a}-s_{b}} A\left(s_{a}\right)+\frac{s-s_{a}}{s_{b}-s_{a}} A\left(s_{b}\right)+\frac{1}{2}\left(s-s_{a}\right)\left(s-s_{b}\right) E(s),
$$

where the matrix $E(s)$ denotes the perturbation matrix in the interpolation. Then instead of solving the usually nonlinear eigenvalue problem (3.1) we make a linear approximation

$$
\left[\frac{s-s_{b}}{s_{a}-s_{b}} A\left(s_{a}\right)+\frac{s-s_{a}}{s_{b}-s_{a}} A\left(s_{b}\right)\right] w=0
$$

which leads to a generalized eigenvalue problem

$$
A\left(s_{a}\right) w=\sigma A\left(s_{b}\right) w
$$

with

$$
\sigma=\frac{s-s_{a}}{s-s_{b}}=1+\frac{s_{b}-s_{a}}{s-s_{b}} .
$$

Therefore, an $s$ value which causes the Jacobian $F_{x}(x(s), s)$ to be singular can be approximated by

$$
\hat{s}=s_{b}+\frac{1}{\sigma-1}\left(s_{b}-s_{a}\right) .
$$

Our approach differs from that in [10] in that the roles of $s_{a}$ and $s_{b}$ are interchanged. The method of [10] solves

$$
A\left(s_{b}\right) \hat{w}=\hat{\sigma} A\left(s_{a}\right) \hat{w}
$$

where

$$
\hat{\sigma}=\frac{s-s_{b}}{s-s_{a}}=1-\frac{s_{b}-s_{a}}{s-s_{a}} .
$$

In the approach of [10], $F_{x}(x, s)$ is factored at $s_{a}$ in order to compute $d x / d s$ for the predictor; that factorization is used again to precondition an Arnoldi iteration for the corrector, and one last time in the formulation of the eigenvalue problem to detect singularities. Since we do not factor $F_{x}$ at all, the roles of $s_{a}$ and $s_{b}$ can be interchanged. We found in our experiments that our approach, using (3.3), gave a better approximation of the location of the singularity that one using (3.6).

Equation (3.4) implies that a bifurcation point $s$ closest to the interval $\left[s_{a}, s_{b}\right]$ corresponds to the largest eigenvalue in magnitude $\sigma$ of (3.3). A negative $\sigma$ signals that there is a bifurcation point between $s_{a}$ and $s_{b}, 0<\sigma<1$ indicates that there is a bifurcation point close behind $s_{a}$, and a "large" positive $\sigma$ means that a bifurcation point is approaching. When $\sigma \approx 1$, it should be interpreted that no bifurcation point is nearby.

Since $\left(x\left(s_{b}\right), s_{b}\right)$ is a regular point and $A\left(s_{b}\right)$ is nonsingular, the generalized eigenvalue problem (3.3) can be solved via the equivalent linear eigenvalue problem

$$
A\left(s_{b}\right)^{-1} A\left(s_{a}\right) w=\sigma w,
$$


where only the largest eigenvalue and corresponding eigenvector are needed. We will solve (3.8) with the Arnoldi method and prove that if $s_{a}$ and $s_{b}$ are not too near a singlar point then the eigenvalue problem is well conditioned in a mesh independent way.

A simple fold point can be predicted in a similar way with $s$ replaced by $\lambda$ and $A(s)$ replaced by $A(\lambda)=G_{u}(u(s), \lambda(s))$.

At a simple bifurcation point two branches of solutions intersect nontangentially. We next describe how the information obtained from the solution of the eigenvalue problem can be used for branch switching. Suppose a bifurcation point $x_{0}=x\left(s_{0}\right)=\left(u\left(s_{0}\right), \lambda\left(s_{0}\right)\right)$ on the primary branch is determined. Since the eigenvector $w$ corresponding to the largest eigenvalue $\sigma$ for the eigenvalue problem described above is an approximation for a null vector of $F_{x}\left(s_{0}\right)$, thus $\left[G_{u}\left(s_{0}\right), G_{\lambda}\left(s_{0}\right)\right] w \approx$ 0 . On the other hand, the tangent vector $\dot{x}_{0}=d x / d s\left(s_{0}\right)$ which is the solution of

$$
\left[\begin{array}{cc}
G_{u}\left(s_{0}\right) & G_{\lambda}\left(s_{0}\right) \\
N_{u}\left(s_{0}\right) & N_{\lambda}\left(s_{0}\right)
\end{array}\right]\left[\begin{array}{l}
\dot{u}\left(s_{0}\right) \\
\dot{\lambda}\left(s_{0}\right)
\end{array}\right]=\left[\begin{array}{c}
0 \\
-N_{s}\left(s_{0}\right)
\end{array}\right]
$$

is also a null vector of $\left[G_{u}\left(s_{0}\right), G_{\lambda}\left(s_{0}\right)\right]$. Since $\operatorname{dim}\left(\mathcal{N}\left[G_{u}\left(s_{0}\right), G_{\lambda}\left(s_{0}\right)\right]\right)=2$ and $w$ and $\dot{x}_{0}$ are linearly independent it is recommended in [10], where $G_{u}$ is factored and $d x / d s$ is computed using (3.9), that $\mathcal{N}\left(\left[G_{u}\left(s_{0}\right), G_{\lambda}\left(s_{0}\right)\right]\right)$ be approximated by $\operatorname{span}\left\{\dot{x}_{0}, w\right\}$. In the matrix-free case considered in this paper, we approximate the tangent vector by a secant approximation

$$
\delta_{x}=\frac{x\left(s_{0}\right)-x\left(s_{-1}\right)}{s_{0}-s_{-1}} .
$$

We approximate the tangent direction of the new branch by orthogonalizing $w$ against $\delta_{x}$

$$
\hat{w}=w-\frac{\delta_{x}^{T} w}{\delta_{x}^{T} \delta_{x}} \delta_{x}, \quad \tilde{w}=\hat{w} /\|\hat{w}\|_{2}
$$

To obtain a regular point on the secondary branch, we solve the following augmented nonlinear system

$$
F_{2}(x)=\left[\begin{array}{c}
G(x) \\
N_{2}(x)
\end{array}\right]
$$

where

$$
N_{2}(x)=\tilde{w}^{T}\left(x-x_{0}-\epsilon \tilde{w}\right)
$$

and $\epsilon$ is a "switching factor". This switching factor is problem dependent and should be chosen large enough so that the solution of (3.11) does not fall back to the primary branch [14]. The nonlinear equation (3.11) can be solved by Newton-type method with initial iterate $x_{0}+\epsilon \tilde{w}$. After moving onto the secondary branch, the continuation procedure for path following on the secondary branch is identical to the one on the primary branch.

In [10] the linear systems for computing tangent vectors (3.9) are solved with a preconditioned Arnoldi iteration and the eigenvalues of the Hessenberg matrix produced in the Arnoldi iteration are used to predict singular points. Since a factorization of $A\left(s_{a}\right)$ is used as the preconditioner in [10], the prediction comes with very little cost. In our approach, Jacobians are not factored and the tangent vector is not computed, instead, a secant vector is used as an approximation. The eigenvalue problem for singularity prediction is performed separately using some iterative 
algorithm. This leaves us the flexibility of choosing any robust iterative solver and the associated preconditioner for the continuation procedure.

Other methods for singularity detection based on solution of eigenvalue problems have been proposed in [25], [9], and [20].

4. Approximations and Mesh Independence. This is the only section in which the properties of the discrete problems are explicitly addressed. We assume, as is standard in the integral equations literature [1], [3], that the discretization used in (1.2) has been constructed, by interpolation if necessary, so that $G^{h}$ has the same domain and range as $G$.

Recall [1] that a family of operators $\left\{T^{\alpha}\right\}_{\alpha \in \mathcal{A}}$ is collectively compact if the set

$$
\cup_{\alpha} T^{\alpha}(\mathcal{B})
$$

is precompact in $X$. In (4.1) $\mathcal{B}$ is the unit ball in $X$. In the special case that the indexing set $\mathcal{A}$ is an interval $\left[0, h_{0}\right]$, we will denote the index by $h$. We say that $T^{h} \rightarrow T$ strongly as $h \rightarrow 0$ and write

$$
T^{h} \stackrel{s}{\rightarrow} T
$$

if $T^{h} u \rightarrow T u$ as $h \rightarrow 0$ in the norm of $X$ for all $u \in X$.

All of the results are based on the following results from [1] and some simple consequences.

THEOREM 4.1. Let $\left\{T^{h}\right\}$ be a family of collectively compact operators on $X$ that converge strongly to $T \in \mathcal{C O} \mathcal{M}(X)$. Assume that $I-T$ is nonsingular. Then there is $h_{0}>0$ such that $I-T^{h}$ is nonsingular for all $h \leq h_{0}$ and $\left(I-T^{h}\right)^{-1}$ converges strongly to $(I-T)^{-1}$.

A simple compactness argument implies uniform bounds for parameter dependent families of collectively compact strongly convergent operators.

COROLLARY 4.2. Let $a<b$ be given and assume that

$$
\left\{T^{h}(s)\right\}_{h \geq 0, s \in[a, b]}
$$

is a collectively compact family of operators such that $T^{h}(s) \stackrel{s}{\rightarrow} T^{0}(s)=T(s)$ for each fixed $s$. Assume that $\left\{T^{h}(s)\right\}$ is uniformly Lipschitz continuous in $s$ and that $I-T(s)$ is nonsingular for all $s \in[a, b]$. Then there are $M, h_{0}>0$ such that

$$
\left\|I-T^{h}(s)\right\| \leq M \text { and }\left\|\left(I-T^{h}(s)\right)^{-1}\right\| \leq M
$$

for all $h \leq h_{0}$ and $s \in[a, b]$.

We let

$$
\Gamma^{h}(\lambda)=\left\{(u, \lambda) \mid G^{h}(u, \lambda)=0, \lambda \in \mathbb{R}\right\} .
$$

We assume that

ASSUMPTION 4.1.

1. $G^{h}(u, \lambda) \rightarrow G(u, \lambda)$ for all $(u, \lambda) \in X \times \mathbb{R}$ as $h \rightarrow 0$.

2. $G^{h}$ is Lipschitz continuously Fréchet differentiable and the Lipschitz constants of $G_{u}^{h}$ and $G_{\lambda}^{h}$ are independent of $h$.

3. For all $(u, \lambda) \in X \times \mathbb{R}$

$$
G_{u}^{h}(u, \lambda) \stackrel{s}{\rightarrow} G_{u}(u, \lambda) \text { as } h \rightarrow 0 .
$$


4. There are $\delta_{0}, h_{0}>0$ such that if

$$
\mathcal{N}(\delta)=\{(v, \mu)|\|v-u\|+| \mu-\lambda \mid<\delta \text { for some }(u, \lambda) \in \Gamma\},
$$

then the families of operators

$$
\left\{I-G_{u}^{h}(v, \mu)\right\}_{\left\{(v, \mu) \in \mathcal{N}\left(\delta_{0}\right), h \in\left[0, h_{0}\right]\right\}} \text { and }\left\{G_{\lambda}^{h}(v, \mu)\right\}_{\left\{(v, \mu) \in \mathcal{N}\left(\delta_{0}\right), h \in\left[0, h_{0}\right]\right\}}
$$

are collectively compact.

We augment $G^{h}=0$ with a mesh-independent Lipschitz continuously differentiable arclength normalization $N(u, \lambda, s)=0$ and defining

$$
F^{h}(x, s)=\left[\begin{array}{c}
G^{h}(x) \\
N(x, s)
\end{array}\right]=0 .
$$

Examples of normalizations $N$ that do not depend on $h$ are (2.6) and (2.7). We let

$$
\Gamma(s)=\left\{(u(s), \lambda(s))|F(u, \lambda, s)=0,| s-s_{0} \mid<\rho, \text { for some } \rho \in \mathbb{R}\right\} .
$$

Consistently with the notation in the previous sections, we let $u^{h}(\lambda)$ and $x^{h}(s)$ denote solutions to $G^{h}(u, \lambda)=0$ and $F^{h}(x, s)=0$.

4.1. The Corrector Equation and Simple Folds. At regular points when $\lambda$ is used as the continuation parameter the equation for the Newton step is

$$
G_{u}^{h}(u, \lambda) z=-G^{h}(u, \lambda)
$$

The theory in [1] asserts that if $G_{u}(u, \lambda)$ is nonsingular, so is $G_{u}^{h}$ for sufficiently small $h$ and $G_{u}^{h}(u, \lambda)^{-1}$ is strongly convergent to $G_{u}(u, \lambda)^{-1}$. However, this convergence is not uniform and in order to invoke the results of [1] and [5] we must take care to remain away from singular points.

One can treat simple folds as regular points by means of arclength continuation. If we solve the augmented system, (2.5), and this system has only regular points, then $\left\|F_{x}^{-1}\right\|$ is bounded on finite segments of $\Gamma$. If, moreover, the normalization $N(u, \lambda, s)$ does not depend on the discretization, as it will not if (2.6) or (2.7) are used, then our assumptions imply that the finite dimensional problems are as well conditioned as the infinite dimensional problems.

THEOREM 4.3. Let Assumptions 1.1 and 4.1 hold. Let $\left[s_{a}, s_{b}\right]$ be such that

$$
\hat{\Gamma}=\left\{\hat{\Gamma}(s): s \in\left[s_{a}, s_{b}\right]\right\} \subset\left\{\Gamma(s): s \in\left[s_{a}, s_{b}\right]\right\}
$$

is a single smooth arc and $F_{x}$ is nonsingular on $\hat{\Gamma}(s)$ then there are $\delta_{1}, K$, and $h_{0}$ such that $\kappa\left(F_{x}^{h}(x, s)\right) \leq K$ for all $(x, s) \subset \mathcal{N}\left(\delta_{1}\right) \times\left[s_{a}, s_{b}\right]$ and $h \leq h_{0}$.

Proof. Assumption 1.1 and the mesh independence of $N$ imply that we may apply Corollary 4.2 to $T^{h}(s)=F_{x}^{h}(x(s), s)$. Hence, there is $h_{0}>0$ and $M$ such that for all $h \leq h_{0}$ and $s \in\left[s_{a}, s_{b}\right]$

$$
\left\|F_{x}^{h}(x(s), s)\right\| \leq M \text { and }\left\|F_{x}^{h}(x(s), s)^{-1}\right\| \leq M .
$$

Let $L$ be the ( $h$-independent) Lipschitz constant of $F_{x}^{h}$. Then if $\|x-x(s)\| \leq \delta \leq \delta_{0}$

$$
\left\|F_{x}^{h}(x, s)\right\| \leq M+L \delta .
$$


Moreover

$$
\left\|I-F_{x}^{h}(x(s), s)^{-1} F_{x}^{h}(x, s)\right\| \leq M L \delta,
$$

and hence if $M L \delta<1$ the Banach Lemma implies that $F_{x}^{h}(x, s)$ is nonsingular and

$$
\left\|F_{x}^{h}(x, s)^{-1}\right\| \leq \frac{M}{1-M L \delta} .
$$

So if $\delta_{1}<1 /(2 M L)$ the proof is complete with $K=2 M\left(M+L \delta_{1}\right)$.

The bound, (4.7), part 2 of Assumption 4.1, and the Kantorovich theorem [11], [15], [17], imply convergence of $x^{h}$ to $x$.

COROLlARY 4.4. Let Assumptions 1.1 and 4.1 hold. Let $\left[s_{a}, s_{b}\right]$ be such that $\{\hat{\Gamma}(s): s \in$ $\left.\left[s_{a}, s_{b}\right]\right\}$ is a single smooth arc with at most simple fold singularities. Then there is a unique solution arc for $F^{h}$,

$$
\hat{\Gamma}^{h}(s) \subset \mathcal{N}(\delta) \times\left[s_{a}, s_{b}\right]
$$

and $x^{h}(s) \rightarrow x(s)$ as $h \rightarrow 0$ uniformly for $s \in\left[s_{a}, s_{b}\right]$.

So, for $h$ sufficiently small, the secant predictor

$$
\left(x^{h}\right)^{(0)}\left(s_{1}\right)=x^{h}\left(s_{0}\right)+\frac{s_{1}-s_{0}}{s_{0}-s_{-1}}\left(x^{h}\left(s_{0}\right)-x^{h}\left(s_{-1}\right)\right),
$$

converges to $x^{(0)}\left(s_{1}\right)$ as $h \rightarrow 0$ uniformly in $\left(s_{-1}, s_{0}, s_{1}\right)$. Hence if the steps in arclength $\left\{\delta_{s}^{n}\right\}$ are independent of $h$ and the secant predictor is used, then the accuracy of the initial iterate to the corrector equation and by Theorem 4.3 the condition of the linear equation for the Newton step is independent of $h$. Hence the performance of the nonlinear iteration is independent of $h$.

As for the GMRES iteration that computes the Newton step, the methods from [16], [4], and [5], may be extended to show that the GMRES iteration for the Newton step converges $\mathrm{r}$ superlinearly in a manner that is independent of $h, x$, and $s$. All that one needs is an uniform clustering of the eigenvalues of $F_{x}^{h}(x, s)$ on $\mathcal{M}(\delta)$ that is independent of $x, s$, and $h$. This follows directly from a theorem in [1]. In the results that follow, we count eigenvalues by multiplicity and order them by decreasing distance from 0 .

THEOREM 4.5. Let $\left\{T^{h}\right\}$ be a family of collectively compact operators on $X$ that converge strongly to $K \in \mathcal{C O} \mathcal{M}(X)$. Let $\left\{\lambda_{j}^{h}\right\}_{j=1}^{\infty}$ be the eigenvalues of $T^{h}$ and $\left\{\lambda_{j}\right\}_{j=1}^{\infty}$ be the eigenvalues of T. Let $\rho>0$ and assume that $\left|\lambda_{j}\right|<\rho$ for all $j>M$. Then there is $h_{0}>0$ such that if $h \leq h_{0}$ then

$$
\left|\lambda_{j}^{h}\right|<\rho \text { and } \lim _{h \rightarrow 0} \lambda_{j}^{h}=\lambda_{j}
$$

for all $1 \leq j \leq M$. Moreover, if $\lambda_{1}$ has algebraic and geometric multiplicity one, then so does $\lambda_{1}^{h}$ for $h \leq h_{0}$. Moreover there is a sequence of eigenfunctions $\left\{w^{h}\right\}$ of $T^{h}$ corresponding to the eigenvalue $\lambda_{1}^{h}$ so that $w^{h} \rightarrow w$, and eigenfunction of T correpsonding to the eigenvalue $\lambda_{1}$.

With this in hand, the eigenvalue clustering result can be obtained in the same way that Theorem 4.3 was derived from Theorem 4.1. We begin with the analog of Corollary 4.2.

COROLlARY 4.6. Let the assumptions of Theorems 4.3 and 4.5 hold. Let $\left\{m u_{j}^{h}(x, s)\right\}_{j=1}^{\infty}$ be the eigenvalues, counted by multiplicity, of $I-F_{x}(x, s)$. Let $\rho>0$ be given. There are $h_{0}, M>0$ such that if $h \leq h_{0}$ then

$$
\left|\mu_{j}^{h}(x(s), s)\right|<\rho
$$


for all $s \in\left[s_{a}, s_{b}\right]$ and $j>M$.

THEOREM 4.7. Let the assumptions of Theorems 4.3 and 4.5 hold. Let $\left\{m u_{j}^{h}(x, s)\right\}_{j=1}^{\infty}$ be the eigenvalues, counted by multiplicity, of $I-F_{x}(x, s)$. Let $\rho>0$ be given. There are $\delta_{2}, h_{0}, M>0$ such that if $h \leq h_{0}$ and $(x, s) \in \mathcal{N}\left(\delta_{2}\right) \times\left[s_{a}, s_{b}\right]$ then $h_{0}, M>0$ such that if $h \leq h_{0}$ then

$$
\left|\mu_{j}^{h}(x, s)\right|<\rho
$$

for all $j>M$.

Before we state our r-superlinear convergence theorem we must set some more notation. We write the equation for the Newton step for the corrector equation as

$$
F_{x}^{h}(x, s) d^{h}=-F(x, s)^{h} .
$$

We let $d_{k}^{h}(x, s)$ denote the $k$ th GMRES iteration and $r_{k}^{h}(x, s)$ the $k$ th GMRES residual. As is standard in nonlinear equations, $d_{0}^{h}=0$, and so $r_{0}^{h}=-F^{h}(x, s)$. The proof of Theorem 4.8 is similar to that of the similar results in [4].

THEOREM 4.8. Let Assumptions 1.1 and 4.1 hold. Let $\left[s_{a}, s_{b}\right]$ be such that $\hat{\Gamma}=\{\hat{\Gamma}(s): s \in$ $\left.\left[s_{a}, s_{b}\right]\right\}$ is a single smooth arc and $F_{x}$ is nonsingular on $\hat{\Gamma}(s)$ Then there are $\delta_{3}>0, h_{1}>0$, and a continuous function $M$ on $(0,1)$ such that for all $\rho \in(0,1)$ and $(x, s) \subset \mathcal{N}\left(\delta_{3}\right) \times\left[s_{a}, s_{b}\right]$ and $h \leq h_{1}$

$$
\left\|r_{k}^{h}(x, s)\right\| \leq M(\rho) \rho^{k}\left\|F^{h}(x, s)\right\| .
$$

Theorem 4.8 states that any desired rate of linear convergence can be obtained in a mesh independent way and hence, for $(x, s)$ fixed, the convergence is r-superlinear in a mesh independent way.

4.2. Simple Bifurcation. Now consider a path $\hat{\Gamma}_{0} \subset \Gamma$,

$$
\hat{\Gamma}_{0}=\left\{\Gamma(s): s \in\left[s_{a}, s_{b}\right]\right\}
$$

which has a single simple bifurcation at $s=s_{c} \in\left(s_{a}, s_{b}\right)$. We let $\hat{\Gamma}_{1}$ be the branch of solutions that intersects $\hat{\Gamma}_{0}$ at $\left(x\left(s_{c}\right), s_{c}\right)$. We will denote solutions on $\hat{\Gamma}_{1}$ by $y$ and the arclength parameter on $\hat{\Gamma}_{1}$ by $t$. Hence, for $t \in\left[t_{a}, t_{b}\right]$,

$$
F(y(t), t)=0 \text {. }
$$

We set $\left(y\left(t_{c}\right), t_{c}\right)$ be the bifurcation point on $\hat{\Gamma}_{1}$. Hence $y\left(t_{c}\right)=x\left(s_{c}\right)$.

For any $\delta>0$, the results in $\S 4.1$ hold for the paths

$$
\begin{aligned}
& \hat{\Gamma}_{0}^{-}=\left\{\hat{\Gamma}_{0}(s): s \in\left[s_{a}, s_{c}-\delta\right]\right\}, \hat{\Gamma}_{0}^{+}=\left\{\hat{\Gamma}_{0}(s): s \in\left[s_{c}+\delta, s_{b}\right]\right\} \\
& \hat{\Gamma}_{1}^{-}=\left\{\hat{\Gamma}_{1}(t): t \in\left[t_{a}, t_{c}-\delta\right]\right\}, \text { and } \hat{\Gamma}_{1}^{+}=\left\{\hat{\Gamma}_{1}(t): t \in\left[t_{c}+\delta, t_{b}\right]\right\}
\end{aligned}
$$

We define $\hat{\Gamma}_{0}^{h}$ in a similar way. Since $x^{h}(s) \rightarrow x(s)$ uniformly for $s \in\left[s_{a}, s_{c}-\delta\right] \cup\left[s_{c}+\delta, s_{b}\right]$ by the results in the previous section, simple bifurcation from $\hat{\Gamma}_{0}^{h}$ can only arise for $s \in\left(s_{c}-\delta, s_{c}+\delta\right)$.

In this section we describe how the prediction of the bifurcation point, the conditioning of the generalized eigenproblem, the solution of that eigenproblem, and the accurate tracking of the other branch depend on $h$. Since perturbation of $G$, even in finite dimension, can change the structure 
of $\hat{\hat{\Gamma}}_{0}$ from two intersecting arcs to two disconnected arcs [18], [19], we cannot show that the bifurcation will be preserved, however if $s_{c}$ is far enough away from $s_{a}$ and $s_{b}$, the new other path will be detected even if it does not correspond to a bifurcation for the finite dimensional problem.

For $(x(s), s) \in \hat{\Gamma}_{0}^{ \pm}, F_{x}(x, s)$ will be nonsingular and we can consider the eigenvalue problems

$$
F_{x}^{h}\left(x^{h}\left(s_{b}\right), s_{b}\right)^{-1} F_{x}^{h}\left(x^{h}\left(s_{a}\right), s_{a}\right) w^{h}=\sigma^{h} w^{h}
$$

where the largest eigenvalue in magnitude is sought. If (4.11) is solved via the Arnoldi method, the matrix-vector products of $F_{x}^{h}\left(x^{h}\left(s_{b}\right), s_{b}\right)^{-1} F_{x}^{h}\left(x^{h}\left(s_{a}\right), s_{a}\right)$ with a vector will each require a product of $F_{x}^{h}\left(x^{h}\left(s_{b}\right), s_{b}\right)^{-1}$ with a vector, $i$. e. a linear solve. If this solve is performed with GMRES, then Theorem 4.8 implies that the number of iterations required to approximate that matrix-vector product can be bounded independently of $h, x, s$.

Our assumptions imply, if $s_{b}-s_{a}$ is sufficiently small, that $\sigma=\sigma^{0}$ is a simple eigenvalue with geometric multiplicity one. Since

$$
\left\{I-F_{x}^{h}\left(x^{h}\left(s_{b}\right), s_{b}\right)^{-1} F_{x}^{h}\left(x^{h}\left(s_{a}\right), s_{a}\right)\right\}_{h \geq 0}
$$

is a collectively compact and strongly convergent sequence, we can apply Theorem 4.5 to conclude that for $h$ sufficiently small, $\sigma^{h}$ is a simple eigenvalue with geometric multiplicity one as well. Moreover $\sigma^{h}$ will be well separated from the next largest eigenvalue in magnitude, hence the conditioning of the eigenvalue problem is independent of $h$.

5. Numerical Results. In the example presented in this section we use the normalization equation

$$
\begin{aligned}
N(u, \lambda, s)= & \theta\left(\frac{u\left(s_{0}\right)-u\left(s_{-1}\right)}{s_{0}-s_{-1}}\right)^{T}\left(u(s)-u\left(s_{0}\right)\right) \\
& +(1-\theta)\left(\frac{\lambda\left(s_{0}\right)-\lambda\left(s_{-1}\right)}{s_{0}-s_{-1}}\right)\left(\lambda(s)-\lambda\left(s_{0}\right)\right)-\left(s-s_{0}\right)=0
\end{aligned}
$$

where $\left(u\left(s_{-1}\right), \lambda\left(s_{-1}\right)\right)$ and $\left(u\left(s_{0}\right), \lambda\left(s_{0}\right)\right)$ are two previously computed solutions and $\theta=0.5$ was used in the experiments. We used a step of

$$
\Delta_{\text {path }}=s_{0}-s_{-1}=.02
$$

in $s$ for path following.

We apply the secant predictor (2.3) to generate the initial iterate. For the corrector, we choose a forward-difference Newton-GMRES algorithm [15]. The outer iteration that generates the sequence $\left\{\left(x^{(k)}, s\right)\right\}$ terminates when the nonlinear residual norm satisfies

$$
\left\|F\left(x^{(k)}, s\right)\right\| \leq \tau_{a}+\tau_{r}\left\|F\left(x^{(0)}, s\right)\right\|
$$

where the absolute error tolerance $\tau_{a}=10^{-7}$ and relative error tolerance $\tau_{r}=10^{-7}$ were used. To avoid oversolving on the linear equation for the Newton step $z_{k}$ the forcing terms $\eta_{k}$ such that

$$
\left\|F_{x}\left(x^{(k)}, s\right) z_{k}+F\left(x^{(k)}, s\right)\right\| \leq \eta_{k}\left\|F\left(x^{(k)}, s\right)\right\|
$$


were adjusted with a method from [8]. We use the $l^{2}$ norm and scale the norm by a factor of $1 / N$ for differential and integral equations so that the results are independent of the computational mesh.

The generalized eigenvalue problem (3.3) that characterizes the singularity prediction and branch switching need not be solved at each new point on the path is computed (i. e. $s_{b}=s_{a}+\Delta_{\text {path }}$ is too frequent). In the example considered in this section, we solved the eigenvalue problem to make a predicition of bifurcation after a step of

$$
\Delta_{\text {eig }}=s_{b}-s_{a}=20 * \Delta_{\text {pred }}=4
$$

had been taken. We used a simple version of the Arnoldi, [2], method based on modified GramSchmidt orthogonalization to solve (3.3) with reorthogonalization at each iteration. The Jacobian of the inflated system is usually neither symmetric nor positive definite, we treat the generalized eigenvalue problem as linear eigenvalue problem and solve the linear system involved explicitly with preconditioned GMRES.

After $j$ steps the Arnoldi method produces

$$
A\left(\lambda_{b}\right)^{-1} A\left(\lambda_{a}\right) Q_{j}=Q_{j} H_{j}+h_{j+1, j} q_{j+1} e_{j}^{T},
$$

where $H_{j}$ is an upper Hessenberg matrix with the $h_{i j}$ 's as its nonzero entries and $Q_{j}$ is orthogonal with the $q_{j}$ 's as columns. If $(\theta, y)$ is an eigenpair of $H_{j}$ and $w=Q_{j} y$, then

$$
\left\|A\left(\lambda_{b}\right)^{-1} A\left(\lambda_{a}\right) w-\theta w\right\|_{2}=\left|h_{j+1, j} \| e_{j}^{T} y\right| .
$$

provides a computable error bound. The Ritz pair, $(\theta, w)$, is used to approximate the eigenpair of $A\left(\lambda_{b}\right)^{-1} A\left(\lambda_{a}\right)$.

We terminated the Arnoldi iteration when

$$
\left|h_{j+1, j}\right|\left|e_{j}^{T} y\right|<10^{-4}
$$

or $j>5$. The prediction $\hat{\lambda}$ is accepted when $\hat{\lambda}$ lies in between $\lambda_{a}$ and $\lambda_{b}$, or

$$
\left|\hat{\lambda}-\lambda_{b}\right|<\frac{1}{2}\left|\lambda_{a}-\lambda_{b}\right| .
$$

We found this strategy sufficient for the example here and were able to use (3.10) to move onto the new branch. Of course, the values of $\Delta_{e i g}$ is problem dependent (as it is for other methods) as is the criteria for accepting a prediction.

As an example we consider the following two-point boundary value problem [14].

$$
\begin{aligned}
& u^{\prime \prime}+u^{3}+\lambda=0 \quad \text { in }(0,1), \\
& u(0)=u(1)=0,
\end{aligned}
$$

Uniform mesh of spacing $h=\frac{1}{N}$ is used to define the grid points $\left\{t_{j}\right\}_{j=0}^{N}$ as $t_{j}=j * h$. A fourth order finite difference discretization leads to the discrete nonlinear system of order $N-1$

$$
\left(\frac{1}{h^{2}}+\frac{1}{12} u_{j-1}^{2}\right) u_{j-1}-\left(\frac{2}{h^{2}}-\frac{5}{6} u_{j}^{2}\right) u_{j}+\left(\frac{1}{h^{2}}+\frac{1}{12} u_{j+1}^{2}\right) u_{j+1}+\lambda=0,
$$


FIG. 5.1. Solution $u\left(\frac{1}{4}\right)$ versus $\lambda$.

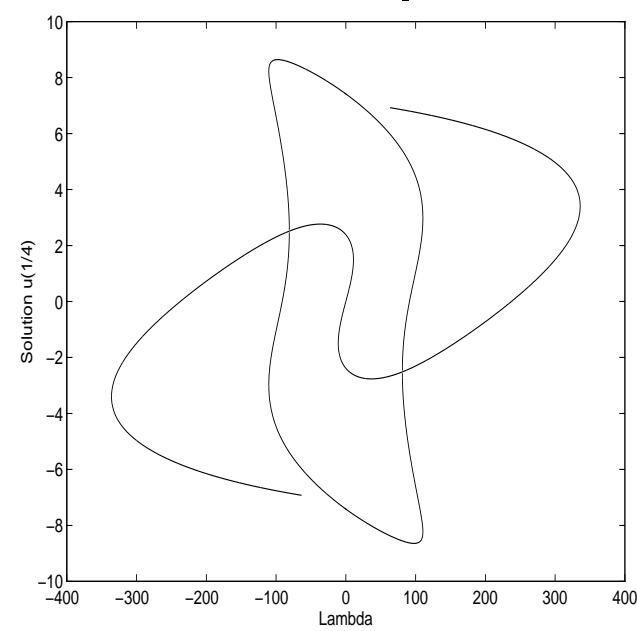

for $j=1, \ldots, N-1 . u_{0}=u_{N}=0$ at the boundaries.

Starting from the trivial solution $u=0$ at $\lambda=0$, the algorithm traces the solution path, locates bifurcation points, switches branches, and captures the secondary solution curves. Indeed there are total two bifurcation points at $\lambda \approx 81,-81$ and eight turning points at $\lambda \approx \pm 11, \pm 110, \pm 336$ in the region of interest. The primary solution branch represents the symmetric solutions and the secondary solution branch represents nonsymmetric periodic solutions bifurcating from the primary branch. Figure 5.1 plots the solution $u\left(\frac{1}{4}\right)$ versus $\lambda$ showing the folds and bifurcations on the curve.

In Table 5.1 we report the bifurcation predictions along the solution path going toward $\lambda \approx$ -81 . One can see that (3.3) is a more accurate predictor than (3.6). Tables 5.2 and 5.3 illustrate the mesh independence of the linear and nonlinear iterations at various points on the primary (5.2) and secondary ((5.3) branches. The iteration statistics remain virtually unchanged as the mesh is refined.

Table 5.4 lists the total number of preconditioned GMRES iterations and Arnoldi iterations corresponding to three representative prediction intervals. In Table 5.4 we show how both the GMRES iterations needed to approximate the product of $F_{x}^{h}\left(x^{h}\left(s_{b}\right), s_{b}\right)^{-1}$ with $F_{x}^{h}\left(x^{h}\left(s_{a}\right), s_{a}\right)$ (or $G_{u}^{-1}$ with $G_{u}$ in the case where we predict a turning point) and the overall number of Arnoldi iterations is independent of the mesh. Each Arnoldi step requires about 12-14 GMRES iterations. This is similar to the numbers listed in Table 5.2 and 5.3. The residual in the table is the Arnoldi residual when the iteration terminates. 
TABLE 5.1

Prediction of bifurcation point along the primary branch going toward $\lambda \approx-81 . N=128$.

\begin{tabular}{|cccc|}
\hline$\lambda_{a}$ & $\lambda_{b}$ & prediction $(3.3)$ & prediction (3.6) \\
\hline $1.0000 \mathrm{e}-02$ & $6.0988 \mathrm{e}+00$ & $9.1417 \mathrm{e}+01$ & $1.2288 \mathrm{e}+01$ \\
$6.0998 \mathrm{e}+00$ & $1.0574 \mathrm{e}+01$ & $1.2614 \mathrm{e}+02$ & $-9.3188 \mathrm{e}+03$ \\
$1.0574 \mathrm{e}+01$ & $8.4208 \mathrm{e}+00$ & $4.0988 \mathrm{e}-01$ & $1.4026 \mathrm{e}+01$ \\
$8.4208 \mathrm{e}+00$ & $1.1361 \mathrm{e}+00$ & $-2.2855 \mathrm{e}+01$ & $-3.5346 \mathrm{e}+01$ \\
$1.1361 \mathrm{e}+00$ & $-7.1699 \mathrm{e}+00$ & $-3.6599 \mathrm{e}+01$ & $-4.7426 \mathrm{e}+01$ \\
$-7.1699 \mathrm{e}+00$ & $-1.5772 \mathrm{e}+01$ & $-4.6063 \mathrm{e}+01$ & $-6.1496 \mathrm{e}+01$ \\
$-1.5772 \mathrm{e}+01$ & $-2.4500 \mathrm{e}+01$ & $-5.3739 \mathrm{e}+01$ & $-8.3880 \mathrm{e}+01$ \\
$-2.4500 \mathrm{e}+01$ & $-3.3295 \mathrm{e}+01$ & $-6.0284 \mathrm{e}+01$ & $-1.0833 \mathrm{e}+02$ \\
$-3.3295 \mathrm{e}+01$ & $-4.2128 \mathrm{e}+01$ & $-6.5987 \mathrm{e}+01$ & $-1.3475 \mathrm{e}+02$ \\
$-4.2128 \mathrm{e}+01$ & $-5.0984 \mathrm{e}+01$ & $-7.0930 \mathrm{e}+01$ & $-1.6373 \mathrm{e}+02$ \\
$-5.0984 \mathrm{e}+01$ & $-5.9854 \mathrm{e}+01$ & $-7.5103 \mathrm{e}+01$ & $-1.9663 \mathrm{e}+02$ \\
$-5.9854 \mathrm{e}+01$ & $-6.8734 \mathrm{e}+01$ & $-7.8384 \mathrm{e}+01$ & $-2.3781 \mathrm{e}+02$ \\
$-6.8734 \mathrm{e}+01$ & $-7.7621 \mathrm{e}+01$ & $-8.0558 \mathrm{e}+01$ & $-3.0825 \mathrm{e}+02$ \\
\hline
\end{tabular}

TABLE 5.2

Total number of Newton and preconditioned GMRES iterations on the primary branch.

\begin{tabular}{|c|ccc|}
\hline problem size & $\lambda$ value & Newton & P-GMRES \\
\hline \multirow{3}{*}{$N=64$} & $\lambda=+1.0895 e+01$ & 5 & 10 \\
& $\lambda=-4.0017 e+01$ & 5 & 12 \\
& $\lambda=-8.0127 e+01$ & 5 & 12 \\
\hline \multirow{3}{*}{$N=128$} & $\lambda=+1.0894 e+01$ & 4 & 7 \\
& $\lambda=-4.0163 e+01$ & 5 & 12 \\
& $\lambda=-8.0386 e+01$ & 5 & 13 \\
\hline \multirow{3}{*}{$N=256$} & $\lambda=+1.0894 e+01$ & 4 & 7 \\
& $\lambda=-4.0223 e+01$ & 5 & 13 \\
& $\lambda=-8.0482 e+01$ & 5 & 12 \\
\hline
\end{tabular}

TABLE 5.3

Total number of Newton and preconditioned GMRES iterations at switching point and on the secondary branch.

\begin{tabular}{|c|lcc|}
\hline problem size & \multicolumn{1}{|c}{$\lambda$ value } & Newton & P-GMRES \\
\hline \multirow{3}{*}{$N=64$} & switching & 7 & 21 \\
& $\lambda=-8.1319 e+01$ & 5 & 12 \\
& $\lambda=-1.0020 e+02$ & 5 & 11 \\
\hline \multirow{3}{*}{$N=128$} & switching & 7 & 22 \\
& $\lambda=-8.1571 e+01$ & 5 & 14 \\
& $\lambda=-1.0015 e+02$ & 5 & 13 \\
\hline \multirow{3}{*}{$N=256$} & switching & 6 & 20 \\
& $\lambda=-8.1262 e+01$ & 5 & 12 \\
& $\lambda=-1.0001 e+02$ & 5 & 11 \\
\hline
\end{tabular}


TABLE 5.4

Total number of preconditioned GMRES and Arnoldi iterations required at different sections on the path when a prediction procedure is performed.

\begin{tabular}{|l|rccccc|}
\hline \multicolumn{1}{|c|}{ path } & \multicolumn{1}{c}{$N$} & $\lambda_{a}$ & $\lambda_{b}$ & P-GMRES & Arnoldi & residual \\
\hline turning & 64 & $7.0814 \mathrm{e}+00$ & $1.0632 \mathrm{e}+01$ & 53 & 5 & $2.9490 \mathrm{e}-02$ \\
point & 128 & $6.0998 \mathrm{e}+00$ & $1.0574 \mathrm{e}+01$ & 50 & 5 & $1.9156 \mathrm{e}-05$ \\
near-by & 256 & $9.1379 \mathrm{e}+00$ & $1.0883 \mathrm{e}+00$ & 55 & 5 & $4.6579 \mathrm{e}-03$ \\
\hline regular & 64 & $-3.1304 \mathrm{e}+01$ & $-4.0215 \mathrm{e}+01$ & 65 & 5 & $8.0766 \mathrm{e}-06$ \\
point & 128 & $-2.4500 \mathrm{e}+01$ & $-3.3295 \mathrm{e}+01$ & 69 & 5 & $3.3738 \mathrm{e}-05$ \\
& 256 & $-3.1175 \mathrm{e}+01$ & $-3.9836 \mathrm{e}+01$ & 71 & 5 & $3.0663 \mathrm{e}-05$ \\
\hline bifurcation & 64 & $-6.7012 \mathrm{e}+01$ & $-7.5953 \mathrm{e}+01$ & 56 & 4 & $9.5183 \mathrm{e}-07$ \\
point & 128 & $-6.8734 \mathrm{e}+01$ & $-7.7621 \mathrm{e}+01$ & 56 & 4 & $1.3263 \mathrm{e}-06$ \\
detected & 256 & $-7.4823 \mathrm{e}+01$ & $-8.3605 \mathrm{e}+01$ & 62 & 4 & $1.3251 \mathrm{e}-06$ \\
\hline
\end{tabular}

Acknowledgments. The authors wish to thank Gene Allgower for making us aware of reference [22], Klaus Böhmer for providing us with a copy, and Alastair Spence for many other pointers to the literature. We are also grateful to Dan Sorensen for his council on the solution of eigenvalue problems.

\section{REFERENCES}

[1] P. M. Anselone, Collectively Compact Operator Approximation Theory, Prentice-Hall, Englewood Cliffs, NJ, 1971.

[2] W. ARNOLDI, The principle of minimized iterations in the solution the matrix eigenvalue problem, Quart. Appl. Math., 9 (1951), pp. 152-163.

[3] K. E. ATKINSON, A survey of numerical methods for Fredholm integral equations of the second kind, SIAM, Philadelphia, 1976.

[4] S. L. Campbell, I. C. F. Ipsen, C. T. Kelley, And C. D. Meyer, GMRES and the minimal polynomial, BIT, 36 (1996), pp. 664-675.

[5] S. L. Campbell, I. C. F. Ipsen, C. T. Kelley, C. D. Meyer, and Z. Q. Xue, Convergence estimates for solution of integral equations with GMRES, Journal of Integral Equations and Applications, 8 (1996), pp. 19-34.

[6] R. Dembo, S. Eisenstat, And T. Steihaug, Inexact Newton methods, SIAM J. Numer. Anal., 19 (1982), pp. 400-408.

[7] E. J. Doedel, Lecture Notes on Numerical Analysis of Bifurcation Problems. Lecture notes from Sommerschule über Nichtlineare Gleichungssysteme, Hamburg, Germany, March 17-21, 1997.

Available by anonmyous ftp to ftp.cs.condordia.ca in pub/doedel/doc/hamburg.ps.Z.

[8] S. C. EISENSTAT AND H. F. WALKER, Choosing the forcing terms in an inexact Newton method, SIAM J. Sci. Comput., 17 (1996), pp. 16-32.

[9] J.-S. GuO, W.-W. LIN, AND C.-S. WANG, Nonequivalence deflation for the solution of matrix latent value problems, Lin. Alg. App., 231 (1995), pp. 15-45.

[10] J. Huitfeldt AND A. RUHE, A new algorithm for numerical path following applied to an example from hydrodynamic flow, SIAM J. Sci. Statist. Comput., 11 (1990), pp. 1181-1192.

[11] L. Kantorovich And G. Akilov, Functional Analysis, Pergamon Press, New York, second ed., 1982.

[12] H. B. Keller, Constructive methods for bifurcation and nonlinear eigenvalue problems, in Computing Methods in Applied Science and Engineering, R. Glowinski and J. Lions, eds., vol. 704, Springer-Verlag, 1977, pp. 241-251.

[13] - Numerical solution of bifurcation and nonlinear eigenvalue problems, in Applications of Bifurcation Theory, P. H. Rabinowitz, ed., Academic Press, 1977, pp. 359-384. 
[14] H. B. KeLLER, Lectures on Numerical Methods in Bifurcation Theory, Tata Institute of Fundamental Research, Lectures on Mathematics and Physics, Springer-Verlag, New York, 1987.

[15] C. T. KELLEY, Iterative methods for linear and nonlinear equations, SIAM, Philadelphia, 1995.

[16] C. T. Kelley And Z. Q. XuE, GMRES and integral operators, SIAM J. Sci. Comput., 17 (1996), pp. 217226.

[17] J. M. Ortega And W. C. Rheinboldt, Iterative Solution of Nonlinear Equations in Several Variables, Academic Press, New York, 1970.

[18] E. RIESS, Column buckling-an elementary example of bifurcation, in Bifurcation Theory and Nonlinear Eigenvalue Problems, J. Keller and S. Antman, eds., W.A. Benjamin, New York, 1969, pp. 1-16.

[19] _ Imperfect bifurcation, in Applications of Bifurcation Theory, P. Rabinowitz, ed., Academic Press, 1977, pp. 37-71.

[20] A. RUHE, Algorithms for the nonlinear eigenvalue problem, SIAM J. Numer. Anal., 10 (1973), pp. 674-689.

[21] Y. SAAD AND M. SCHULTZ, GMRES a generalized minimal residual algorithm for solving nonsymmetric linear systems, SIAM J. Sci. Statist. Comput., 7 (1986), pp. 856-869.

[22] R. Sebastian, Andwendung von Krylov-Verfahren auf Verzweigungs-und Fortsetzungsprobleme, $\mathrm{PhD}$ thesis, Philipps-Universität Marburg, Marburg, Germany, 1995.

[23] H. F. WALKER, An adaption of Krylov subspace methods to path following problems, Tech. Rep. 12/96/88, Mathematics and Statistics Department, Utah State University, December 1996.

[24] _ An approach to continuation using Krylov subspace methods, Tech. Rep. 1/97/89, Mathematics and Statistics Department, Utah State University, January 1997.

[25] Y. F. ZHOU AND A. RUHE, Numerical path following and eigenvalue criteria for branch switching, in Large Scale Eigenvalue Problems, J. Cullum and R. Willoughby, eds., North-Holland, 1986, pp. 319-334. 